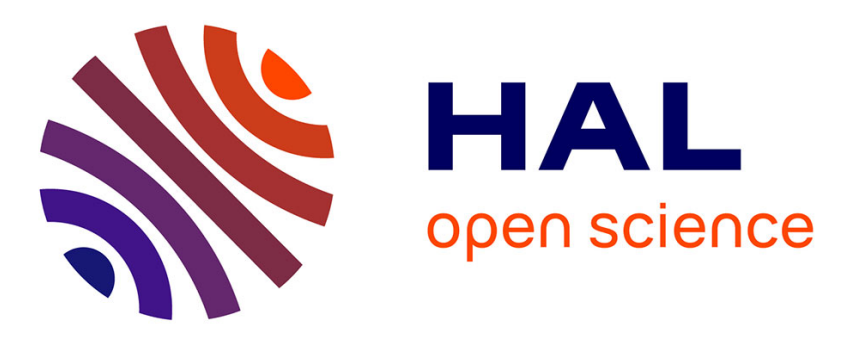

\title{
Time-resolved quantum transport for optoelectronics
}

Fabienne Michelini, Katawoura Beltako, M. Bescond, N. Cavassilas, L. Raymond

\section{To cite this version:}

Fabienne Michelini, Katawoura Beltako, M. Bescond, N. Cavassilas, L. Raymond. Time-resolved quantum transport for optoelectronics. 2017 IEEE International Electron Devices Meeting (IEDM), Dec 2017, San Francisco, France. 10.1109/IEDM.2017.8268478 . hal-01761825

\section{HAL Id: hal-01761825 \\ https://hal.science/hal-01761825}

Submitted on 15 May 2018

HAL is a multi-disciplinary open access archive for the deposit and dissemination of scientific research documents, whether they are published or not. The documents may come from teaching and research institutions in France or abroad, or from public or private research centers.
L'archive ouverte pluridisciplinaire HAL, est destinée au dépôt et à la diffusion de documents scientifiques de niveau recherche, publiés ou non, émanant des établissements d'enseignement et de recherche français ou étrangers, des laboratoires publics ou privés. 


\title{
Time-resolved quantum transport for optoelectronics
}

\author{
F. Michelini, K. Beltako, M. Bescond, N. Cavassilas and L. Raymond \\ Aix Marseille Univ, Univ Toulon, CNRS, IM2NP UMR 7334, Marseille, France, email: fabienne.michelini@im2np.fr
}

Abstract - We investigate time-resolved energy currents in a molecular optoelectronic junction made of two donors and an acceptor sandwiched between two electrodes and excited by a Gaussian femtosecond laser pulse. Features of direct energy currents are thus correlated to the intra-molecular structure.

\section{INTRODUCTION}

Recent experimental trend toward femtosecond multidynamical excitations has triggered an interest for investigating time-dependent regimes in nanostructured semiconductor, molecular or hybrid electronic systems [1]. Molecular electronic devices form indeed a promising alternative to standard electronic devices due to their fast response on ultra-short time scale. The quest for understanding the time-resolved response of nanometer scale junctions interacting with external electromagnetic fields is thus fueled by both the fundamental questions it raises and the premise for long term applications in optoelectronics. In this delicate context, the problem of energy transfer is emerging as one of the most crucial issues at several levels, from the impasses meat by quantum thermodynamics [2] to biologically inspired high efficiency concepts [3]. In this paper, we derive and examine time-resolved energy currents induced by femtosecond laser pulses in a molecular optoelectronic junction sandwiched between metallic electrodes.

\section{THEORETICAL ORIENTATIONS}

By the end of the last century, nanodevices reached the nanometer scale, and lived a tremendous change of paradigm as they become quantum systems. Such a breakthrough has revealed powerful methods of quantum statistics for simulating nanowire transistor characteristics, in particular the method of non-equilibrium Green's functions (NEGFs) [4]. Today, this formalism is widely used for several device functionalities, but challenges remain, especially for timedependent regimes and quantum thermoelectricity.

Few years ago, a technique was proposed by Gaury and co-workers in order to manage time-dependence in mesoscopic systems [5]. Elementary objects of the technique are wave functions (WFs) instead of NEGFs, vectors instead of matrices. In fact, WFs are components of the retarded Green's function projected on propagation modes that are defined from reservoir self-energies. Despite being applied only in non-interacting cases so far, the technique seems also promising to tackle interactions. The technique aims to manage time-dependence by means of simpler simulations while accounting for the complete energy dependence of the self-energies, i.e. beyond the wide band limit.
On the other side, quantum thermoelectricity has recently emerged as a part of the solution to the problem of sustainable emission-free energy production. However, these advances have raised some fundamental questions about energy and heat [6], which are crucial for thermodynamics and energy conversion at the nanoscale especially in time-dependent regimes [2]. Morevor, few teams around the world develop specific time-resolved techniques to directly address the photo-carrier dynamics [1], and even less the photo-carrier thermodynamics at the nanometer scale.

We present a practical methodology based on lattice Hamiltonian to investigate energy exchanges on ultimate time and size scales. The approach is developed for the study of a molecular optoelectronic junction that interacts with femtosecond laser pulses.

\section{ENERGY CURRENT IN THE MOLECULAR JUNCTION}

We consider an optoelectronic junction made of three subsystems: two donors $\left(\mathrm{D}_{1}\right.$ and $\left.\mathrm{D}_{2}\right)$ interact with a Gaussian femtosecond laser pulse, and they are both connected to an acceptor (A). The whole complex is in contact with two thermal reservoirs. The junction is shown Fig. 1. Each donor is described by a two-level model of HOMO and LUMO states. For the acceptor, we use a two-site model with a single level model to study energy transport. All parameters are shown in the molecular complex level structure of Fig. 2.

For the loop circuit designed by the molecular complex, see Fig. 3, we derive an energy current operator from the continuity equation. In Ref. [7], the authors developed an energy current operator for a one-dimensional lattice Hamiltonian. We here address the loop case. The difficulty underlying the definition of an energy current operator resides in the fact that in contrast with the charge current operator for which: $N=\sum_{s} n_{s}$, the total Hamiltonian of the system doesn't have the form of a sum of subsystem Hamiltonians. Indeed $H=\sum_{s} h_{s}^{0}+\sum_{r(s)} V(s, r):$ all sites $s$ have a local Hamiltonian, $h_{s}^{0}$, but they are also interconnected with coupling terms $V(s, r)=t_{s r} a_{s}^{+} a_{r}+h . c$., where $t_{s r}$ is the coupling parameter, $t_{s r}=0$ if sites $s$ and $r$ are not connected.

We have to redefine local Hamiltonians that include intersite coupling terms. We adopt the same proposal as [7] with $h_{s}=h_{s}^{0}+\frac{1}{2} \sum_{r(s)} V(s, r)$. 
For this peculiar circuit, we identify four contributions to energy current operator by comparison with the onedimensional discrete case treated by $\mathrm{Wu}$ and Segal [7]. This viewpoint makes possible to distinguish between the two single line terms, two-site $J^{E(2)}$ and four-site $J^{E(4)}$ terms, the additional branch term, $J^{E(B)}$, and finally two loop contribution in $J^{E(L)}$. All these contributions are schematically represented Fig. 3. Time-resolved mean values of these contributions and total energy current were calculated using WF technique [5], still in the wide band limit.

\section{FINDINGS}

In Fig. 4, the time-resolved energy current exhibits oscillations whose frequency varies as a function of the $D_{1}-D_{2}$ coupling. These oscillations manifest the proper frequencies of the internal structure of the molecular complex. Indeed, the donor-donor coupling modifies the internal structure of the complex: frequencies change. As long as electrons have enough time to explore all the hybridized states in the system, they oscillate between these states before tunneling to reservoirs. To study the effects of couplings and excitation characteristics, we now rely on the direct energy current, that is the energy current integrated over time.

The direct energy current together with its different components are plotted as a function of the $\mathrm{D}_{1}-\mathrm{D}_{2}$ coupling in Fig. 5. The $J^{E(2)}$ term represents the two-site current which is directly proportional to charge current. This contribution is the major one. The $J^{E(4)}$ term represents the four-site contribution along the D1-A line, while $J^{E(B)}$ involves the $\mathrm{D}_{2}$-A branch. However, term $J^{E(B)}$ is several orders of magnitude lower than $J^{E(4)}$ because carriers cannot reach the left reservoir via this branch (tunneling parameter is zero for left LUMO states). Finally, the loop term, $J^{E(L)}$, provides a negative contribution to the total energy current. The $D_{1}-D_{2}$ coupling drives energy in the loop formed by the two donors and the acceptor. Increasing simultaneously both D-A and $\Gamma$ couplings naturally emphasize both $J^{E(4)}$ and $J^{E(L)}$ contributions (see Fig. 3 for schematic definitions).

We finally explore impacts on energy current of a dissymmetry between the two donors.

The first difference is introduced via excitation. We suppose that the two laser pulses are not synchronized on a femtosecond timescale, a delay occurs between the two lightdonor interactions. For this study, two molecule configurations are considered: the case where the donors are not connected, and the loop in its highly symmetric configuration, where the two donors are connected with a coupling parameter equal to the D-A coupling value, both cases are shown Fig. 6. While a monotonous behavior of both currents is observed in the first case, in the loop case the two currents flowing from $\mathrm{D}_{1}$ and $\mathrm{D}_{2}$ towards $\mathrm{A}$ are oscillating with an opposite phase and a frequency about D-A coupling.
The second difference between the two donors is implemented in the D-A coupling parameter. The $\mathrm{D}_{2}-\mathrm{A}$ coupling value increases from zero to twice the $\mathrm{D}_{1}$-A one. In the $\mathrm{D}_{1}-\mathrm{A}$ branch, energy current decreases while it increases starting from zero in the $\mathrm{D}_{2}-\mathrm{A}$ branch, as plotted Fig. 7. Interestingly, the sum of the two currents exhibits a maximum close to the symmetric configuration also shown Fig. 7.

At last, the roles of D-D and D-A couplings are exchanged: the $D_{2}-A$ coupling is zero while the $D_{1}-D_{2}$ coupling increases from zero to twice the $\mathrm{D}_{1}$-A coupling value. We observe two remarkable behaviors on Fig. 8. On the one hand, $J_{D 1-A}^{E}$ increases when $\mathrm{D}_{1}$ is connected to $\mathrm{D}_{2}$, and on the other hand, $J_{D 2-A}^{E}$ is non-zero while the two sites $\mathrm{D}_{2}$ and $\mathrm{A}$ are not connected. This intriguing last result is originating from one of the $J^{E(L)}$ components. Indeed, from the definition we have chosen, $J^{E(L)}$ includes a term which is not proportional to the coupling between $\mathrm{D}_{2}$ and $\mathrm{A}$ (see Fig. 8). What is the meaning of this contribution? We believe that it is likely related to transport channels activated by the time-dependent electromagnetic field. Otherwise, the energy current operator shall be accordingly adapted.

\section{CONCLUSION}

We studied the dynamics of energy transfer at donor-acceptor interfaces in a molecular optoelectronic junction using a mean-field approach inside the wave function technique. This exploratory work still ignores electron-electron interaction and vibrations, but nonetheless provides basic insights towards the design of new energy-conversion schemes for time-dependent optoelectronics.

\section{ACKNOWLEDGMENT}

The authors acknowledge financial support from the ANRFrench National Research Agency [NOODLES, contract No. ANR-13-NANO-0009].

\section{REFERENCES}

[1] A. A. Bakulin, C. Silva, and E. Vella, Ultrafast Spectroscopy with photocurrent detection: Watching Excitonic Optoelectronic Systems at work", J. Phy. Chem. Lett. 7, pp. 250-258, 2016.

[2] M. Esposito, “The nature of heat:”, Phys. Rev. B , pp (2015).

[3] C. Creatore, M. A. Parker, S. Emmott, and A.W. Chin, "Efficient biologically insired photocell enhanced by delocalized states”, Phys. Rev. Lett. 111, 253601, 2013.

[4] G. Stefanucci and R. van Leeuwen, "Nonequilibrium Many-Body Theory of Quantum Systems: a Modern Introduction”, Cambridge: Cambridge University Press, 2013.

[5] B. Gaury, J. Weston, M. Santin, M. Houzet, C. Groth, and X. Waintal, "Numerical simulations of time-resolved quantum electronics" Phys. Rep. 534(1): pp. 1- 37, 2014.

[6] F. Michelini, A. Crépieux, and K. Beltako, "Entropy production in photovoltaic- thermoelectric nanodevices from the non-equilibrium Green's function formalism", J. Phys.: Condens. Matter 29, 175301, 2017.

[7] L.-A. Wu and D. Segal, "Energy lux operator, current conservation and the formal Fourrier's law”, J. Phys. A: Math. Theor. 42, 025302, 2009. 


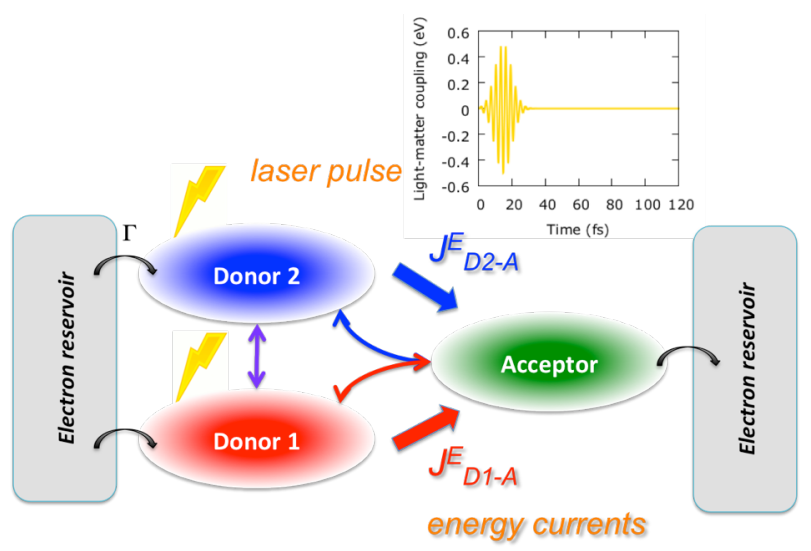

Fig. 1. Schematics of the molecular complex junction. The Gaussian pulse has a central frequency equal to the donor gap $(1.4 \mathrm{eV})$, a width of $30 \mathrm{fs}$ and an amplitude of $1 \mathrm{eV}$.

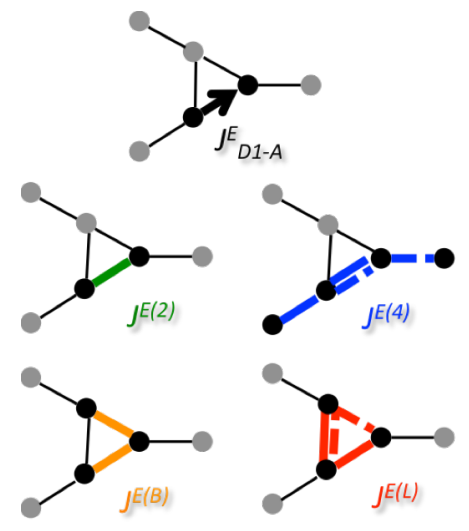

Fig. 3. Molecular loop circuit. The four contributions to the energy current flowing from $\mathrm{D}_{1}$ to $\mathrm{A}$. Colored lines represent the inter-site couplings involved in each contribution.

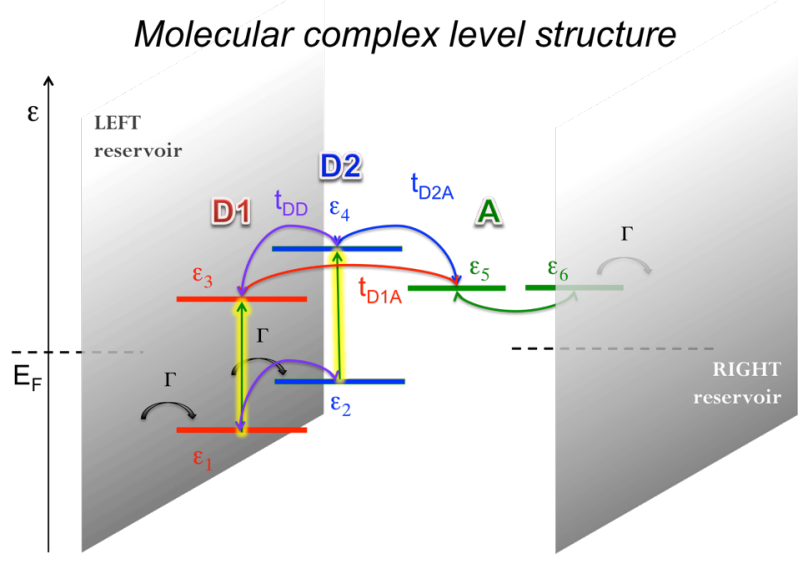

Fig. 2. Molecular level structure and set of parameters. By-default configuration is given by: all inter-site couplings $\mathrm{t}_{\mathrm{ij}}$ are equal to $0.2 \mathrm{eV}$ (including coupling to reservoir $\Gamma$ ), and all gaps are equal to $1.4 \mathrm{eV}$.

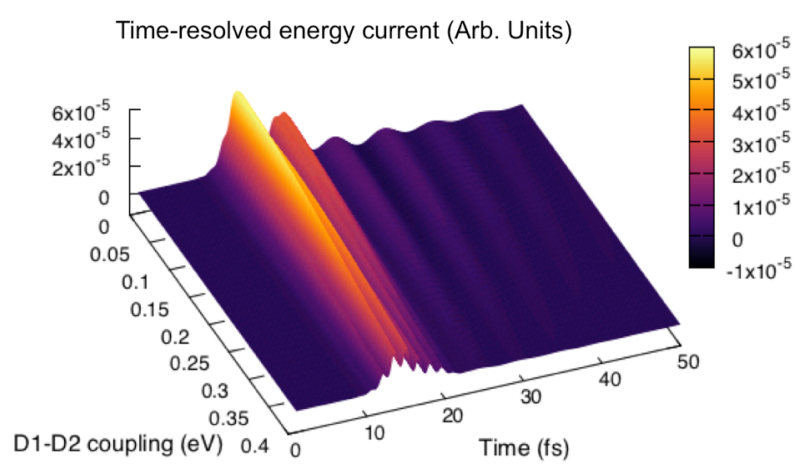

Fig. 4. Time-resolved energy current as a function of the donordonor coupling.
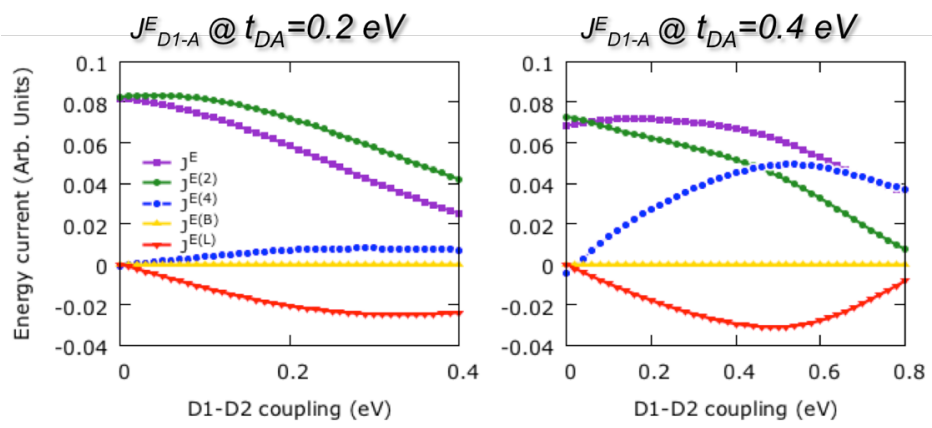

Fig. 5. Direct energy current as a function of the donor-donor coupling for two values of D-A coupling. All components of energy current are shown. 

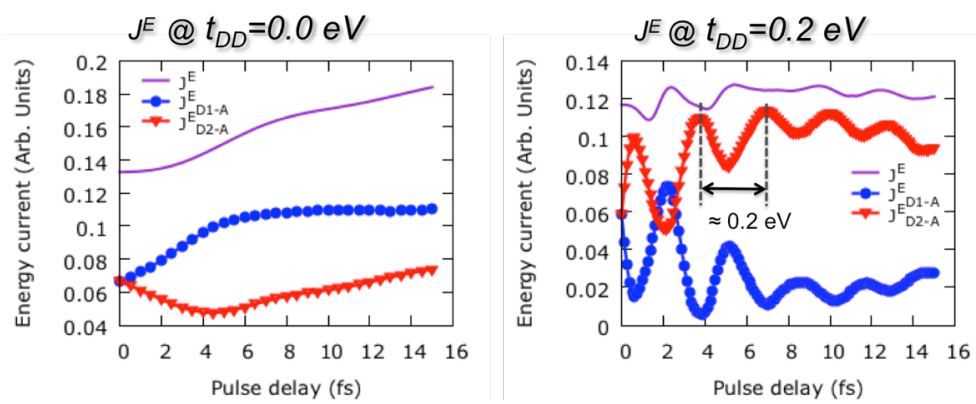

Fig. 6. Direct energy current as a function of the delay between the two femtosecond laser pulses for two D-D coupling values.
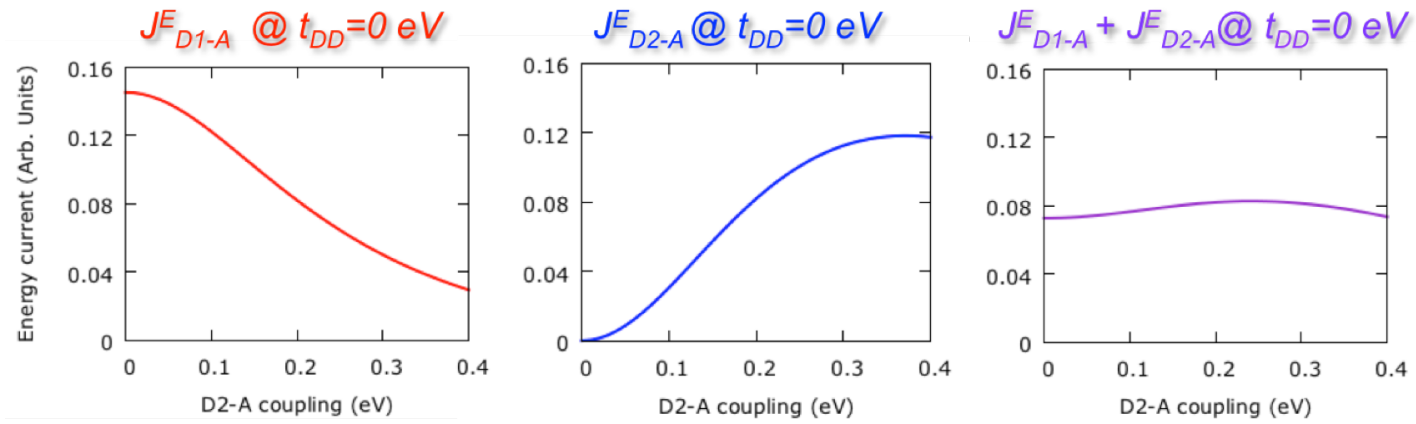

Fig. 7. Direct energy currents, $J_{D 1-A}^{E}, J_{D 2-A}^{E}$ and their sum, as a function of the $\mathrm{D}_{2}-\mathrm{A}$ coupling.
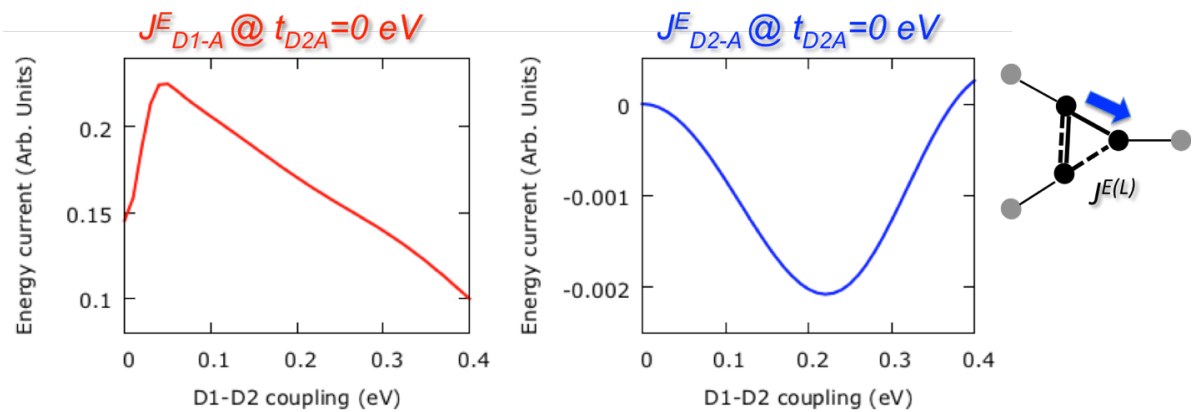

Fig. 8. Direct energy current as a function of the D-D coupling when sites $\mathrm{D}_{2}$ and $\mathrm{A}$ are disconnected. Schematics of inter-site couplings involved in the $J^{E(L)}$ component of $J_{D 2-A}^{E}$. 